\title{
$94{ }^{\mathrm{e}}$ Congrès de l’AOCS (Kansas City, 4-7 mai 2003) L'essentiel du programme Nutrition
}

Oléagineux, Corps Gras, Lipides. Volume 10, Numéro 5-6, 333-7, Double 5-6, SEPTEMBRE-OCTOBRENOVEMBRE-DÉCEMBRE 2003, NUTRITION

Auteur(s) : François MENDY, Conseiller Scientifique, 2, rue du Calvaire, 92210 Saint-Cloud, France .

\section{ARTICLE}

\section{Auteur(s) : François MENDY}

Conseiller Scientifique, 2, rue du Calvaire, 92210 Saint-Cloud, France

Malgré l'ambiance un peu étrange de ce congrès, en partie dépendante des événements extérieurs, le succès habituel des congrès de l'AOCS ne s'est pas démenti. Plus de 600 communications techniques ou scientifiques permettaient ainsi de se confronter à une évolution qui ne cesse jamais. Pourtant certaines années sont plus que d'autres des années de crise ou de transition. C'est un chercheur au double passé industriel et universitaire, Mark Matlock, qui a été élu président de I'AOCS. Son discours rappelant l'exigence d'une absence d'interférence entre des responsabilités dans la "gouvernance " de l'AOCS, et des fonctions industrielles et commerciales, la nécessité de dissocier absolument ces deux activités, était étonnant. En plus de 20 ans d'AOCS, je n'en avais jamais entendu l'équivalent. Certes, je connaissais l' « antitrust policy " de l'AOCS comme tous les membres, mais le besoin de le rappeler aussi nettement, m'a paru intéressant, et à vrai dire approprié. Enfin, quelques anciens membres du Conseil de " Gouvernance » ont été nommés " AOCS Fellows » et semblent avoir été placés, peut-être, en situation d'attente ou d'honorariat comme YS. Huang, ex-Président de la Division Health and Nutrition. Cette année, la Division Health and Nutrition avait choisi trois thèmes pour ce congrès :

- "The slimmed-down version of the national academy of sciences DRI (dietary reference intakes) macronutrient report... just the fats » et les CLAs en nutrition humaine.

- Lipid oxydation and oxydative stress in vivo.

- Un sujet de "protéinomique »: "The emerging role of dairy proteins and bioactive peptides in nutrition and health ».

The slimmed-down version of the national academy of sciences DRI macronutrient report... just the fats

Ce rapport a en fait été diffusé par l'US Institute of Medicine (IOM) sous l'égide de l'Académie des Sciences le 5 septembre 2002. A sa sortie, il déclencha de très vives réactions : "Please don't tell me to die faster " W.E.M. Lands (Senior Scientific Advisor NIH) Inform 13 Décembre 2002, 896. II avait été critiqué également, de façon indirecte, par W. Harris, le cardiologue de Kansas City, et par ailleurs Vice-Président du comité de nutrition de l'american heart association. (Am J Clin Nutr 2003 ; 77 : 27980). Cette journée devait donc être le cadre d'une confrontation argumentée, pertinente. La 
direction de l'AOCS en a décidé semble-t-il autrement. Le programme a été changé pour un programme de "paix", sans confrontation. Au comité de direction de la Division Health and Nutrition, il a été souligné, qu'un vrai programme serait mis en place pour 2004 et 2005 (et d'ores et déjà une journée : "Composition en acides gras sériques et pathologies diverses " était proposée pour 2004). Ni W.E.M. Lands, ni W. Harris (pourtant de Kansas City), ni S. Cunnane, ni H. Sprecher ne sont venus assister à cette journée à Kansas City, journée qui s'est transformée en simple matinée. Deux communications sont finalement à retenir.

- Challenges in developping DRI (dietary reference intakes) and macronutrients

P. Kris-Etherton (Présidente du comité de nutrition de l'american heart association)

Cette communication est en fait une analyse très neutre du rapport de l'Académie des sciences :

- I'Al (Adequate Intake) du linoléique est fixé à $17 \mathrm{~g} / \mathrm{j}$ pour les jeunes hommes, $12 \mathrm{~g} / \mathrm{j}$ pour les femmes (" median intake ... where the presence of deficiency is non-existant »), soit autour de $6 \%$ de l'A.E.T.

- l'Al (Adequate Intake) de $\alpha$-linolénique est fixé à 1,6 g/pour les hommes, 1,1 g pour les femmes (" median intake ... where the presence of deficiency is non-existant »), soit autour de $0,6 \%$ de l'énergie (dans la Lyon Diet Heart Study l'apport est de $2 \mathrm{~g} / \mathrm{j}, 0,81 \%$ en.)

- « Trans fatty acids are not essential. SFA, Trans fatty acids, and dietary cholesterol should be as low as possible while consuming a nutritionally adequate diet... ".

- « The Acceptable Macronutrient Distribution Range (AMDR) for FAT is 20 to $35 \%$ en. for adults ».

- « The AMDR for linoleic acid is 5 to $10 \%$ energy ».

- « The AMDR for $\alpha$-linolenic acid is 0,6 to $1,2 \%$ energy ».

- « Higher intakes of $\alpha$-linolenic, EPA, and DHA may afford some degree of protection against CHD ».

- «...It is not possible to estimate one AMDR for All n-3 fatty acids. Up to 10 percent of the AMDR can be consumed as EPA and/or DHA ! " soit $110 \mathrm{mg}$ à $160 \mathrm{mg} / \mathrm{jour}$.

Dans une étude très récente chez des prématurés (31 s. \pm 2 ) Preterrn Infant Formula supplementation with $\alpha$-linolenic acid and docosahexaenoic acid (A. Roddguez, D. Raederstortf, P. Sarda, C. Lauret, F. Mendy and B. Descomps, Eur J of Clin Nutr 2003 ; 57 : 727-34) nous avons obtenu des résultats jamais obtenus jusqu'ici, pratiquement optimun, égaux sur tous les plans au lait maternel (avec ALA $=0,6 \%$ en. ; EPA + DHA (r.1/5) =0,13\% en. ; EPA + DHA/ALA = 22 \%). Ces apports sont très proches de ceux préconisés par le DRI Fats Report de l'Académie des sciences des USA. Par contre, les données de Juin 2003 de l'Afssa, ou celles de la législation européenne de Novel Foods autorisant l'huile de poisson (5 juin 2003) sont très éloignées.

$\mathrm{Ce}$ rapport est ainsi très en retrait : - des souhaits de beaucoup de scientifiques qui pensent qu'un rapport linoléique/ $\alpha$-linolénique supérieur à 6/1, au maximum 8/1, est dangereux, or il autorise des rapports de 10/0,6 ; - des souhaits de certains industriels de l'agro-alimentaire d'utiliser en abondance l'huile de lin en alimentation humaine (malgré le Norwegian Oil Experiment, 1965-1966, $(n=13000)$, Scand J Clin Lab 
Invest 1968; 105 (suppl.): 1-20, qui utilisa l'huile de lin pour un apport hors des limites physiologiques de 5,5 g d'ALA/jour, et dont les résultats catastrophiques firent sombrer les études

sur l'a-linolénique pour plusieurs années);

- des souhaits de groupes industriels comme Roche, Marteck, les producteurs d'huile de poisson et des utilisateurs comme les cinq grands qui se sont engagés à les utiliser comme Mead-Johnson, Ross Products, WYETH et dernièrement Nestlé, après la décision C (2003) de la Commission européenne du 5 juin 2003 autorisant I'utilisation de I'huile de DHA dans des aliments et des boissons... Mais la position de l'Académie de sciences des USA, se base sur la montée en force d'un courant " not disturb physiology " (Voir : " Genetic Defenses Against Non-Cholesterols Stérols », de E.L. Klett, in Current Opinion in Lipidology, August 2003, 14, 4). Ce courant est d'ailleurs ancien. Certes, il n'avait pu faire obstacle à l'excès des AGPI en n-6 préconisé entre 1970 et 1985, mais la suite des événements lui avait donné raison. La position de W.E.M. Lands est défendable, et d'ailleurs le comité met l'accent surtout sur l' $\alpha$-linolénique, même s'il omet le rapport fondamental linoléique/ $\alpha$ linolénique. Par contre les diverses positions exprimées sur l'huile de poisson, plus récemment celle de la CEE, du rapport Afssa, soulèvent de vrais problèmes, et plus encore l'étude SU. FOL. OM3. II est donc normal de voir apparaître dans I'Am J Clin Nutr (Juillet $2003 ; 78$ : 1-2), un éditorial de P. Marckmann (Roskilde Htal, Denmark) : "Fishing for Heart Protection ", soutenant un travail : "n3 fatty acids and 5-years risks of death and cardiovascular disease events in patients with coronay adery disease ", AT Erkkilaetal, Kupio Univ. Finland, and USDA Human Nutr. Res., Tufts Univ. Boston (Am J Clin Nutr $2003 ; 78: 65-71$ ).

Trois avertissements sont clairement formulés dans ces articles : - « For patients with stable angina, there is no benefit from eating fish, and there may even be harmful effects of consumming fish oil capsules » P. Marckmann.

- « In my opinion fish-oil supplementation cannot be recommended for the general population » $P$. Marckmann.

- " ALA, EPA, and DHA are nutritional factors that could potentially reduce the risk of death in patients with CAD. Furthermore this benefit can be obtained through the intake of foods and without intake of supplements ", AT. Erkkila et al. (Les travaux de cette équipe ont été réalisés avec de l'huile de Colza).

Ces travaux confirment de plus l'intérêt exceptionnel du dosage d'acides gras au niveau des esters de cholestérol. En fait, devant la " bulle » syllogistique, favorable et opportuniste, qui s'est constituée autour des huiles de poisson et des acides gras à chaîne très longue, égale ou supérieure à C20, hautement insaturés (HUFA), entraînant "marketing ", technologues, financiers, chercheurs manquant de crédits, la réaction de l'Académie des sciences des USA est légitime. Pour éviter les drames de la sortie de "Bulle Techno " (cf. Norwegian Oil Study, 1965-1966), la meilleure solution est de revenir " aux fondamentaux ».

Deux notions ne peuvent être contournées :

- l'acide gras en n-3 indispensable et essentiel est l'a-linolénique en sn-2, son métabolisme est sévèrement ciblé et contrôlé. Les conditions d'un métabolisme adéquat doivent être étudiées et réunies ; 
- ses deux dérivés supérieurs l'EPA, et encore plus le DHA, sont le fruit d'une synthèse rigoureusement ciblée et contrôlée. Hors du domaine pharmaceutique, toute utilisation en dehors d'apport très limités, et sous une forme de phospholipide en sn-2, est dangereuse (syndrome de Barth, cardiolipines anormales, déstabilisation de plaques athéroscléreuses, transformation d'angines de poitrine stables en angines de poitrines instables, peroxydations explosives...). S'il est un domaine ou le principe de sécurité doit s'appliquer, c'est bien celui-là.

- Conversion of linolenic acid to $n-3$ Icpufa metabolites: estimates and regulation E.A. Emken

Le problème posé par cette communication très intéressante est qu'il s'agit, chez l'homme, d'études d'acides gras libres deutérés, or l'acide gras libre n'est pas réellement un nutriment habituel et lorsqu'il s'agit d'un $\alpha$-linolénique, s'il est libéré en milieu intestinal, son destin est surtout d'être préférentiellement oxydé, car il n'est plus protégé par une situation en sn-2 qui le rend non libérable. En fait ciblé pour une utilisation non énergétique par cette situation particulière, il sera ou métabolisé en dérivés supérieurs ou libéré par la LCAT pour estérifier une molécule de cholestérol. De ce fait, tout le monde attend les résultats des études de R. Brenna de Comwell, réalisées avec des triglycérides ou des phospholipides contenant l'acide gras deutéré étudié en sn- 2 .

Deux phrases sont à retenir :

- " In some studies, n-6 and n-3 enriched diets were used to show that substrate, product, and competitive inhibition are clearly important mechanisms that influence conversion ". Le rapport LA/ALA est fondamental. Lorsqu'il passe de $5 / 1$ à $8 / 1,50 \%$ de la transformation de ALA en EPA ne se fait plus. En fait suivant les conditions choisies le rapport de conversion de ALA en EPA + DHA peut tomber de $17,3 \%$ à $0,2 \%$.

- «Blood lipid composition data for vegetarians and n-3 deficient patients infused with $18: 3 n-3$ (Etude princeps de Holman) suggests conversion of dietary $18: 3 n-3$ to $22: 6 n-3$ is adequate to meet needs ". La Lyon Diet Heart Study, en apparence de prévention secondaire, était bâtie sur l'étude princeps de Holman de façon à analyser ce qui se passait en situation d'apport physiologique en $\alpha$ linolénique en sn-2.

\section{CLA in human nutrition}

Beaucoup d'argent et de temps ont été dépensés sur ce sujet, aboutissant à des erreurs graves en raison de manque de connaissances de biochimie fondamentale pour certains. Ainsi, une "Bulle Techno » est née. D’où pour le comité de l'Académie des sciences des USA : « ...Trans fatty acids are not essential... ", intervention guère sélective.

Deux communications sont à retenir sur les huit présentées:

- "The role of $\Delta 9$-desaturase and endogenous synthesis of conjugated linoleic acids in ruminants", D.E. Bauman, Cornell Univ. II devient de plus en plus évident que la source majeure de l'acide ruménique (9cis-11 trans) est le vaccénique $18: 1,11$ trans, provenant du rumen, désaturé en $\eta-9$ par une $\Delta 9$ désaturase. Cet enzyme est trés sensible à l'insuline. 
- «Application of deuterium-labeled fatty acids for investigation of metabolism and endogenous synthesis ot CLA in humans", H. Adiot and E.A. Emken. Deux données curieuses sont à retenir :

- « The 11-trans isomer was found to be converted to 9 cis-11 trans CLA at a rate 10-fold higher than the 11-cis isomer to 9 cis-11 cis $18: 2 »$.

- "Accumulation of 9c, $11 \mathrm{t}-18: 2$ vs. $10 \mathrm{t}, 12 \mathrm{c}$ in the various plasma lipid classes varied widely by class (9 à $73 \%$ ) and by isomer ... $4 \times$ higher incorporation of $10 \mathrm{t}, 12 \mathrm{c} 18: 2$ in 1 acylphosphatidylcholine ... 2-3 $\times$ higher incorporation of 9 c,11 t-18: 2 vs. 10 t, 12 c: 2 in cholesterol esters ".

Il était étrange de voir consacrer autant de temps aux CLAs qu'aux DRI macronutriments report... just fats. II paraît fort probable que la "bulle » va éclater. II restera l'acide vaccénique, et l'acide ruménique et comme critère biochimique principal, le pourcentage d'acide ruménique (c9, t11-CLA) dans les esters de cholestérol. Une amélioration importante d'une technique d'analyse " Détermination of c9, t11-CLA in human lipid plasma lipid classes with a combination of methylation techniques ». A.M. Shahin et al. (Lipids $2003 ; 38: 793-800$ ) et la possibilité de centrer le travail sur les esters de cholesterol du sérum, vont pouvoir faire progresser efficacement les études cliniques (figures 1 et 2).

\section{Lipid oxydation and oxydative stress in vivo}

C'est la troisième fois que ce thème était repris à l'AOCS, mais jamais il n'avait été traité à un tel niveau de compétence. D'une certaine façon, c'était aussi un éclairage important sur le sujet non réellement traité la première journée. Le mérite de cette journée revient à K.M. Schaich de la Rutgers University, qui a pu réunir onze communications de très haut niveau, et à quelques intervenants dans la salle, vieux routiers de ce sujet (Frankel...).

- Dietary oxidized lipids-implications in atherosclerosis (Emory Univ. Atlanta)

S. Parthasarathy et al.

En 1985, le premier travail de ce chercheur fit l'effet d'une bombe. C'était l'époque du tout linoléique. Actuellement, S. Parthasarathy pense que les acides gras oxydés facilitent la solubilisation du cholestérol luminal, augmentant l'« uptake » endothélial de cholestérol et pouvant conduire ainsi à l'hypercholestérolémie et à l'athérosclérose. II a donc pu démontrer sur des souris " knock-out » récepteur LDL, que l'acide gras oxydé $13-H O D E$ (forme la plus simple du linoléique oxydé), en présence d'un apport diététique en lipides et en cholestérol normal, augmente de $200 \%$ les lésions aortiques, et cet effet est cholestérol indépendant.

- Role of vitamin E bioavailability and Metabolism in Heart Disease M.G. Traber

C'est M.G. Traber qui découvrit en 1995 la protéine-ligand spécifique de l'a-tocophérol. L' $\alpha$ tocophérol et le $\gamma$ sont de puissants antioxydants lipophiles. Le $\alpha$-tocophérol peut de plus " scavenger " les espèces réactives d'azote. Le nitro- $\gamma$-tocophérol (NGT) peut être dosé in vivo. MG. Traber vient de plus de mettre en évidence chez 10 hommes et 6 femmes que la demi-vie du $\gamma$ tocophérol deutéré est de $14 \pm 1 \mathrm{~h}$, vs $40 \pm 3 \mathrm{~h}$ pour l' $\alpha$-tocophérol deutéré. Des études sont en cours 
pour évaluer la production de NGT (produit d'oxydation) et de $\alpha$-CEHC, de $\gamma$-CEHC (métabolites et non produits d'oxydation de l' $\alpha$ et du $\nu$-tocophérol). Ceci permettrait de déterminer si la rapide disparition du $\gamma$-tocophérol deutéré résulte d'un métabolisme ou d'une consommation accrue.

- Antioxydants clinical trials, I. Jialal (UC Davis medical Center) ${ }^{1}$

L' $\alpha$-tocophérol a été montré capable de décroître la peroxydation lipidique, d'inhiber l'adhésion plaquettaire, d'inhiber l'agrégation et la prolifération des cellules musculaires lisses, d'exercer des effets anti-inflammatoires sur les monocytes, d'améliorer la fonction endothéliale. I. Jialal a fait une revue extensive des diverses études sur ce sujet et montre les résultats conflictuels, même si la majorité des résultats semble montrer un bénéfice réel en prévention secondaire. II apparaît de façon claire que des nouvelles études cliniques sont nécessaires avec des méthodologies plus rigoureuses, pour arriver à un consensus (ubiquinone Q10 et variation au niveau du recyclage de l' $\alpha$ tocophérol oxydé ?).

- In vivo effects of dietary lipid peroxydes: a historical overview K.M. Schaich (Rutgers University)

Personne n'était aussi habilité que KM. Schaich pour cet exposé. Dans une première partie, elle a rappelé cette longue histoire qui va de 1960 à 1980, de Kummerow, Kubow, Yagi, Frankel et Tappel, et a établi la toxicité des hydroperoxydes lipidiques. De 1980 à 1990, le discours se précisa, et l'on parla des radicaux hydroxyl, des anions superoxydés, comme espèces créant des dommages biologiques. Beaucoup en sont encore restés à cette période. Mais c'est alors que K.M. Schaich sut analyser l'extraordinaire rapidité des réactions radicalaires et la rapidité des cinétiques de diffusion jusqu'à un arrêt. Ceci ouvrait la voie à de nouvelles identification des rôles des lipides dans le "Cell signaling ", certains processus pathologiques et le rôle des antioxydants, prophylactiques, mais aussi arrêt de signaux. L'objet de ce symposium qu'elle a organisé est de jeter un nouveau regard sur le rôle des peroxydes lipidiques in vivo, dans les phénomènes de peroxydation, de fonctionnement cellulaire (membrane, structure et fonction) dans certains processus pathologiques. L'année précédente GF. Gibbons avait fait un exposé analogue, rappelant les travaux prémonitoires de Kandutsch et Chen, montrant les rôles non du cholestérol mais des oxystérols confirmés par G. Shroepfer plus tard. Ainsi, certains oxystérols sont les ligands pour la transcription du Liver Xreceptor $\alpha$ (LXR $\alpha$ ), de la Sterol Regulatory Element binding protein (SREBP), qui contrôlent avec l'insuline la synthèse endogène de cholestérol et des lipides.

- Mechanisms contributing to formation of elevated levels of modified Idl in the postprandial state F. Ursini, A. Sevandian, (University of Padova et University of South California)

Ce travail est extrêmement important, les lipides oxydés présents dans les aliments sont absorbés (mais aussi les peroxydes lipidiques qui se forment dans le milieu intestinal). Le taux d'hydroperoxydes lipidiques plasmatiques des aldéhydes dérivés s'élève, augmentant (après un passage dans les chylomicrons, les VLDLs, et une arrivée au niveau des LDLs) la susceptibilité à l'oxydation des LDLs. II apparaît dans le plasma des particules LDLs électronégatives, dont on sait qu'elles jouent un rôle dans l'évolution de l'athérosclérose et que l'on sait maintenant doser. En fait, les hydroperoxydes modifient la structure secondaire des apo B. II apparaît aussi très tôt des lysophospholipides dangereux par eux-mêmes. 
- Lipid peroxy radicals: its biochemical effects and scavengers in dietary components H. Maedaa Kanazawa et al. (Kuamamoto University)

Au cours d'un remarquable travail sur la diffusion des espèces radicalaires LOO* et ROO* de l'effet de NO*, les auteurs ont découvert un nouveau composé de l'huile de colza, le 4-vinyldimethoxyphénol, analogue décarboxylé de l'acide sinapique, qui est un très puissant scavenger de LOO*. II bloque le clivage ou les mutations du DNA induites par LOO*. II inhibe aussi l'effet du peroxonitrique (ONOO-) qui est un très puissant oxydant in vivo.

- Effect of diet on lipoprotein oxidation, determination of the level and the structures of oxidized lipoprotein TAGis by base-line-diene conjugation and HPLC-ESI-MS, J-P. Suomela et al. (University of Turku, Finlande)

Les auteurs ont utilisé cette technologie assez lourde pour l'étude des LDLs chez l'homme. Au terme d'une étude chez le porc d'une huile de tournesol avec 3 niveaux d'oxydation différents, ils ont pu établir une grille semi-quantitative des niveaux d'oxydations de différentes lipoprotéines et des structures moléculaires des TAGs oxydés.

Les trois communications suivantes ont traits à des techniques d'ores et déjà accessibles en recherche clinique et qui commencent à être bien documentées.

- The isoprostanes: Their use as an index of lipid per oxidation in vivo, J. Morrow (Vanderbilt University)

C'est J. Morrow qui a découvert les isoprostanes, il y a une dizaine d'années. Dans son remarquable exposé, il a décrit sa découverte des F2-Isoprostanes, produites indépendamment des cyclooxygénases, par une peroxydation radicalaire de l'acide arachidonique in situ sur le phospholipide. Une masse actuellement substantielle de données permet d'affirmer que ces composés sont des indices pertinents du niveau de peroxydation lipidique in vivo. J. Morrow a détaillé toutes les techniques de dosage utilisables.

- Electron paramagnetic resonance spectroscopy in analysis of lipid oxidation N. Parinandi (the Ohio State University, Columbus)

C'est l'exposé qui a suscité le plus d'enthousiasme, tant cette méthode est efficace aussi bien sur le plan qualitatif que quantitatif in vivo pour l'étude de la formation de radicaux lipidiques. Grâce à C. Motta, cette technique est actuellement utilisée en France dans une étude réalisée chez les moines de l'abbaye de Randol et sera secondairement utilisée au cours de l'étude Linolenage d'Olivier Henry, réalisée en gérontologie.

- Amazing antibodies: exquisitely specific tools for analysis of lipid-derived in vivo protein modification

R.G. Salomon et al.

Les isolevuglandines (isoLGs) produits d'oxydation générés par des réarrangements des Isoprostanes Endoperoxydes sont extrêmement réactifs et révélables en tant qu'additions covalentes à des protéines. On est en train de montrer que les niveaux de ces "Isolg-Proteins Adducts " sont un facteur de risque indépendant pour les maladies cardio-vasculaires. Alors que la demi-vie des 
isoprostanes est courte en raison d'un métabolisme rapide et d'une excrétion urinaire, les isoLGs adhèrent immédiatement à des protéines et peuvent s'accumuler pendant des jours et des semaines, permettant ainsi une " dosimétrie » pertinente du stress oxydatif in vivo chez l'homme. Des études immunologiques ont révélés la formation de 2-pentyl pyrroles, 2-carboxyheptyl pyrroles et 2-carboxypropyl pyrroles (CPP) par le biais de la réaction de protéines avec les $\gamma$-hydroxy- $\alpha, \beta$ insaturés aldéhydes produits de l'oxydation lipidique. Des anticorps viennent d'être selectionnés et produits qui présentent une préférence 1000 fois supérieure pour une liaison avec les 2carboxyethylpyrroles (CEP) versus les CPP. Or, les CEP sont les produits uniques de l'oxydation des lipides contenant du docosahexaenoïque (C22: 6n-3 ou DHA). De hauts niveaux de CEP ont été détectés dans les protéines de la rétine d'individus présentant une dégénérescence maculaire de la rétine (AMD) liée à l'âge, comparé avec une cohorte d'individus du même âge sans maladie de la rétine. Les niveaux sanguins moyens de CEP étaient significativement $(p=0,02)$ mais modestement plus élevés que ceux de la cohorte de comparaison. Tous les individus présentant à la fois un taux élevé de CEP et d'auto anticorps CEP faisaient parti de la cohorte AMD. Ces observations impliquent fortement une réponse antiCEP immune dans l'étiologie de l'AMD, et ouvrent ainsi la voie à une pathologie immune médiée par les produits d'oxydation lipidiques liés à des protéines. Le marché que représentent ces kits de dosage de CEP, anti-CEP, CPP, anti-CPP et leur facilité d'utilisation dans des laboratoires de niveau moyen vont très probablement bouleverser ce domaine dans les cinq ans à venir. Ce n'est certainement pas le moment d'élever artificiellement les taux de DHA dans le régime par utilisation d'huiles de poisson ou d'éthyles d'acides gras extrait de l'huile de poisson, ou même de triglycérides reconstitués à partir d'acides gras en C22 : n-3. En terme de protection, ceci n'a rien à voir avec la position en sn-2 des phospholipides de la chair de poisson (ainsi que des antioxydants qu'elle contient).

${ }^{1}$ A paraître dans Circulation 2003.

\section{The emerging role of dairy proteins and bioactive peptides in nutrition and health}

Pendant six ans de 1984 à 1990, un programme de biotechnologie franco-québécois (codirigé par J. Amiot et $\mathrm{F}$. Mendy) s'est consacré à ce sujet. La conjonction entre un universitaire français qui pensait pouvoir récupérer l'ensemble du projet et une grande industrie agro-alimentaire qui souhaitait l'enterrer eut pour effet de précipiter la fin de ce projet. L'arrivée de Bruce German, en provenance de Davis, au centre de recherche de Nestlé à Vevey, semble en faire maintenant un sujet important pour Nestlé. De même, l'arrivée de PJ Huth, membre de l'AOCS, du comité de direction de la Health and nutrition division, à la direction scientifique du national dairy council, a permis la réalisation de cette journée. En France, J-L. Maubois, G. Brulé, L. Roger, P. Guilloteau, A. Rerat et F. Mendy, sur le plan biologique, avaient fait office de précurseurs mais tout ce travail reçut peu d'échos. Pourtant des brevets, témoins des résultats originaux obtenus, ont été pris. Et quelques idées très débattues ont été confirmées à Laval (Québec) pour réapparaître sous forme de produits ou brevets. Aussi pour nous, la plupart des communications présentées manquait quelque peu de nouveautés à l'exception de deux, dont l'une probablement importante pour les spécialistes des lipides.

- The role of dairy proteins and amino acids in the regulation of food intake GH. Anderson et al. (University of Toronto, Canada) 
Les auteurs ont attiré l'attention sur l'effet du glycomacropeptide, produit d'hydrolyse digestive de la caséine kappa sur la sécrétion de la cholecystokinine. La sécrétion de la cholecystokinine est normalement déclenchée par l'ingestion de matières grasses et contribue à la régulation des ingestats dans le sens d'une diminution. Une action combinée semble donc possible.

- The ability of caseinophosphopeptides and casein hydrolysate to inhibit lipid oxydation in oil-inwater emulsions M. Diaz, D.J. McClements and E.A. Decker (MIT Department of Food Science) (Honored Student Award Winner)

Cette communication était présentée par la division Lipid oxydation and quality. M. Diaz a présenté la même communication au congrès de l'ISF à Bordeaux. Ce travail a été soutenu par Bruce German et financé par Nestlé. L'objectif non déguisé est d'essayer d'obtenir un brevet, mais le brevet Brule G, Roger L, Fauquant J et Piot M. (US Patent $N^{\circ} 4358465,1982$ ) obtenu par un travail réalisé avec un financement Sopharga, vient de tomber dans le domaine public et antériorise tout travail en ce domaine. Pour comprendre l'intérêt de cette communication, il faut la relier à celle de F. Ursini, sur l'augmentation des LDL oxydés en post-prandial (cf. infra). Le programme de M. Diaz est exactement le programme que nous voulions lancer avec Mme le Professeur C. Ferradini, à la Faculté de Médecine des Saints-Père et l'appui du Prof. P. Pottier au CNRS en 1990. Les caséino-phosphoprotéides sont la meilleure façon de capter en milieu intestinal, les métaux de transition qui sont les pro-oxydants majeurs de beaucoup d'aliments incluant des émulsions type huile-dans l'eau. De plus (G. Brulé), le phosphopeptide principal, commun avec le lait maternel, est capable de fixer le fer ferrique $(+++)$ et de le réduire en fer ferreux $(++)$, de le protéger dans la traversée intestinale, et de le céder ensuite à une protéine d'absorption sous forme ferrique. Il en est de même pour le cuivre.

"Thus, antioxidant properties might not be uniquely attributed to chelating metals by phosphoseryl residues but also by scavenging free radicals » M. Diaz.

Pour C. Ferradini, on ne pouvait imaginer système plus efficace, complémentaire de la vitamine $C$ et des vitamines et facteurs liposolubles. Pour nous aider, en 1990, l'équipe du Professeur P. Pottier au CNRS, construisit les formules tridimensionnelles du phosphopeptide le plus riche de possibilité, GLUSERP-LEU-SERP-SERP-SERP-GLU-GLU, celui présent également dans le lait maternel, de façon à tenter de comprendre les mécanismes entrant en jeu (figure 3).

\section{Conclusion}

Bien d'autres communications et posters auraient pu être relevés mais il m'a paru absolument nécessaire de relater de la façon la plus claire possible, les orientations majeures qui semblaient se profiler. Dans le cadre de la nutrition, il paraît, enfin, de plus en plus difficile de s'éloigner, de mépriser les régulations physiologiques. La fonctionnalité ne peut s'exercer que dans le cadre de la résilience de l'élasticité et des régulations physiologiques. Un autre élément, celui de la biodisponibilité dépendant de la position en sn-2 sur le triglycéride et/ou le phospholipide, suscite de plus en plus d'études :

- « Post-prandial changes in the positional distribution of fatty acids in VLDLs triacyglycerols » KM. Yli-Jokipii et al., Univ. Turku, Univ. Kuo, Finland (Honored Student Award Winner and Nutrition 
Division Student Excellence Award Winner) : la composition postprandiale des TAGs des VLDLs dépend fortement du poids moléculaire et de la position des acides gras sur le triglycéride alimentaire. Etude réalisée chez l'homme $(n=9)$.

- «Effect of triacyglycerol structure on absorption and metabolism of isotope labeled palmitic and linoleic acids by humans " E. Emken et al. USDA, Illinois St. Univ. La conversion du $18: 2 n-6$ en ses métabolites supérieurs était 2,8 fois plus importante dans le régime lard randomisé supplémenté que dans le régime lard natif supplémenté.

Ainsi, position en sn-2 sur le triacyglycérol, mais aussi position en sn-2 d'un acide gras saturé particulier, le myristique, ( $\mathrm{H}$. Dabadie, et $\mathrm{F}$. Mendy), jouent sur la transformation des acides gras en $\mathrm{n}-6$ et en $\mathrm{n}-3$, en leurs dérivés supérieurs, mais aussi sur leur oxydabilité. La composition en moles des acides gras des esters de cholestérol est en passe de devenir le critère principal de toute étude bioclinique. Or elle est aussi très liée à la position en sn-2 des acides gras des phospholipides et à leur affinité compétitive pour la LCAT. II faut aussi sûrement féliciter l'AOCS pour la création d'un nouveau poster symposium "Teaching innovations in lipid science " dont il faut souhaiter qu'il perdure. L'AOCS vit et contribue à l'effervescence des données dans le domaine des lipides. Controverse, avance trop rapide, critique et remise dans le courant physiologique y sont possibles. On ne peut que souhaiter que son exemple soit suivi.

Illustrations

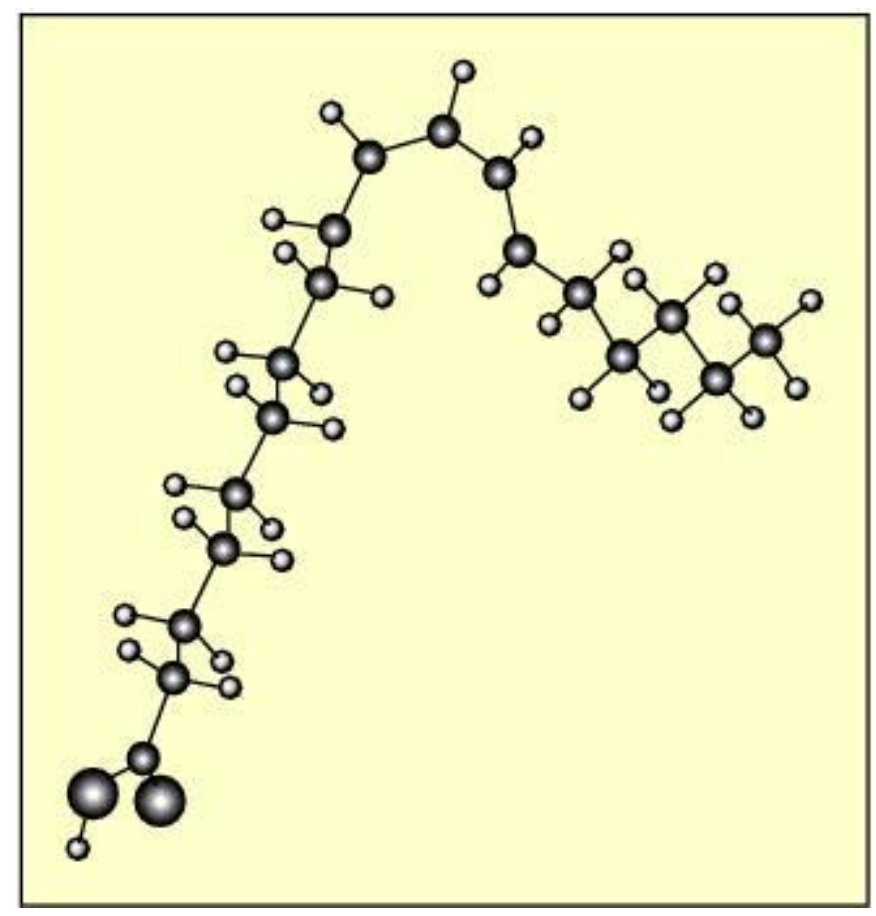

Figure 1. Acide ruménique (C18:2n-7t, 9cis, 11 trans) dans l'eau (une des conformations possibles). Calcul Clotilde Policar. 


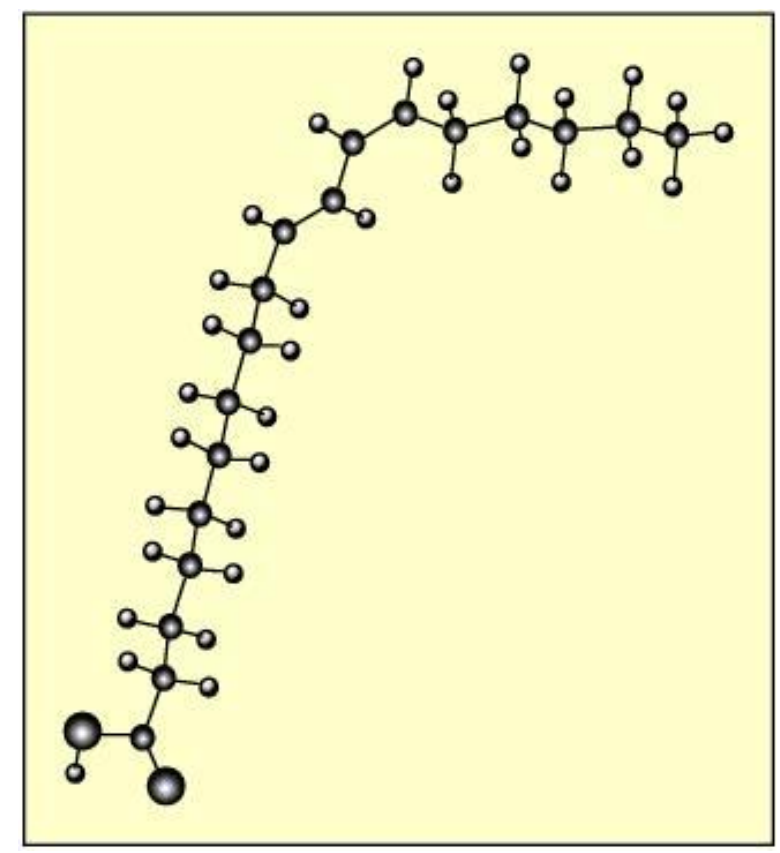

Figure 2. Isomètre lot 12 CLA dans l'eau (une des conformations possibles). Calcul Clotilde Policar.

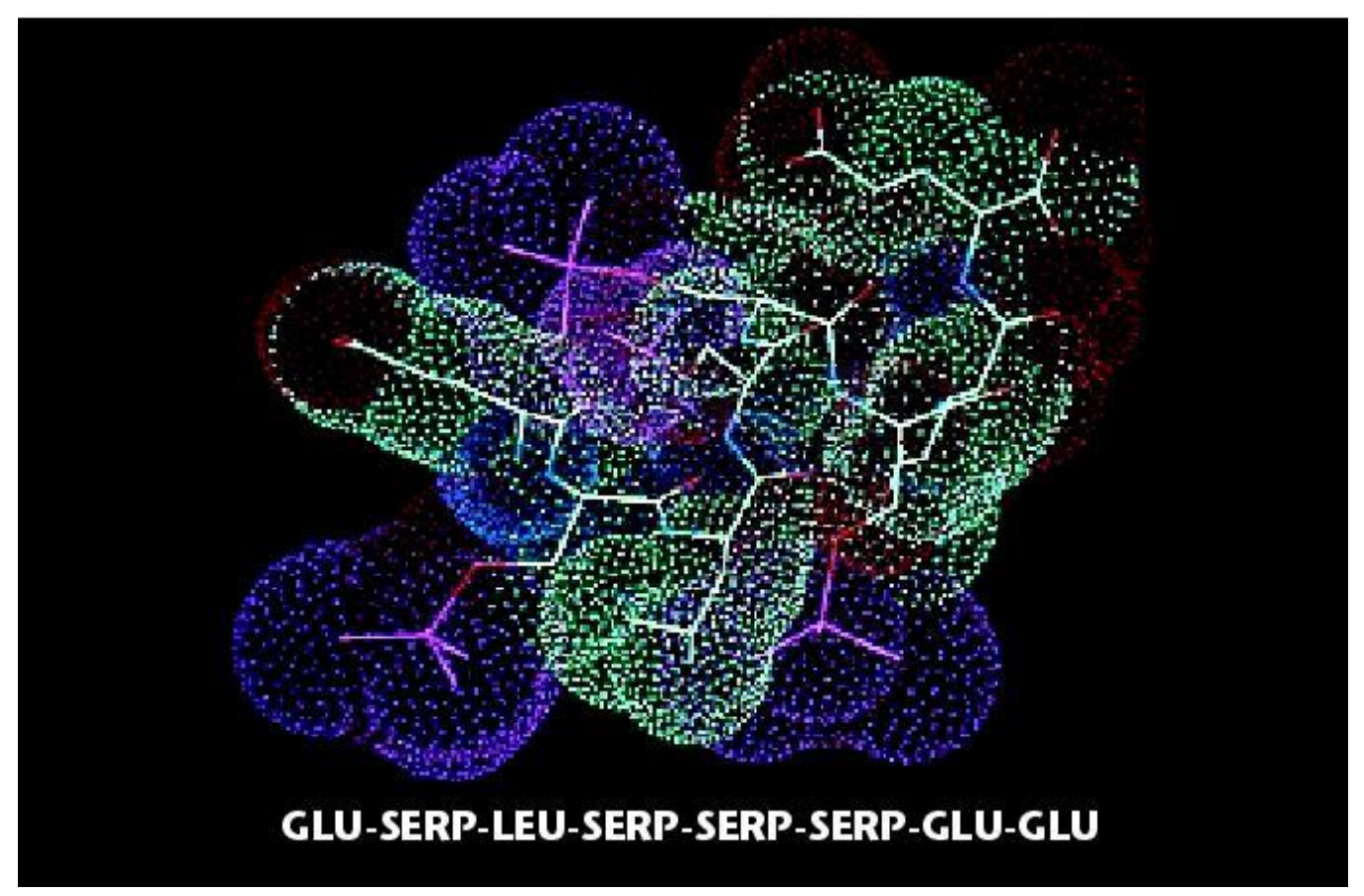

Figure 3. Une des formes possibles du peptide GLU-SERP-LEU-SERP-SERP-SERP-GLU-GLU, calculée, commun au lait de femme et au lait de vache, par l'équipe du Pr. P. Pottier, lab. des substances naturelles, CNRS, Gif-sur-Yvette, à la demande de F. Mendy.

Comprendre la formation des LDL post-prandiales électro-négatives, à partir des lipides oxydés ingérés et/ou des peroxydes lipidiques formés dans le milieu intestinal et absorbés, la prévenir, est certainement un sujet très important. 